\title{
Recording seismic reflections using rigidly interconnected geophones
}

\author{
C. M. Schmeissner*, K. T. Spikes ${ }^{\ddagger}$, and D. W. Steeples ${ }^{\ddagger}$
}

\begin{abstract}
Ultrashallow seismic reflection surveys require dense spatial sampling during data acquisition, which increases their cost. In previous efforts to find ways to reduce these costs, we connected geophones rigidly to pieces of channel iron attached to a farm implement. This method allowed us to plant the geophones in the ground quickly and automatically. The rigidly interconnected geophones used in these earlier studies detected first-arrival energy along with minor interfering seismic modes, but they did not detect seismic reflections. To examine further the feasibility of developing rigid geophone emplacement systems to detect seismic reflections, we experimented with four pieces of channel iron, each $2.7 \mathrm{~m}$ long and $10 \mathrm{~cm}$ wide. Each segment was equipped with 18 geophones rigidly attached to the channel iron at $15-\mathrm{cm}$ intervals, and the spikes attached to all 18 geophones were pushed into the ground simultaneously. The geophones detected both refracted and reflected energy; however, no significant signal distortion or interference attributable to the rigid coupling of the geophones to the channel iron was observed in the data. The interfering seismic modes mentioned from the previous experiments were not detected, nor was any $P$-wave propagation noted within the channel iron. These results show promise for automating and reducing the cost of ultrashallow seismic reflection and refraction surveys.
\end{abstract}

\section{INTRODUCTION}

Near-surface seismic reflection methods have proven useful at depths of $\leq 10 \mathrm{~m}$ (Baker et al., 1999, 2001), thus allowing the depths at which seismic and ground-penetrating radar (GPR) studies are done to overlap in a useful manner. One advantage of GPR is its ability to produce high-quality, high-resolution subsurface images in an economical way. Its disadvantages relative to seismic reflection include strong GPR signal attenuation in clay-rich soils and limited penetration depth.

To bring the imaging power of near-surface seismic reflection methods to the level offered by GPR, the subsurface must be sampled at a significantly greater spatial density and frequency bandwidth than is usual for shallow surveys. However, dense sampling (i.e., geophone intervals of 5 to $15 \mathrm{~cm}$ ) can increase the cost of a near-surface seismic survey greatly. To reduce such costs, we examined the feasibility of planting a large number of geophones rapidly and automatically.

Previous work (Steeples et al., 1999a,b) demonstrates that seismic data can be recorded successfully when geophones are attached rigidly to a piece of wood or to a piece of channel iron. Attaching 72 geophones to five channel-iron bars connected to an agricultural tillage tool allowed all of the geophones to be planted in approximately $2 \mathrm{~s}$. The studies revealed that the rigid attachment of the geophones to a length of channel iron did not affect their performance as detectors of shallow $P$-wave refractions. However, two important questions emerged from those initial experiments: first, whether geophones rigidly mounted to channel iron could also record seismic reflections and, second, whether the source of an anomalous mode of wave propagation found in an earlier study (Steeples et al., 1999b) could be isolated and examined.

\section{METHODS}

\section{Experimental procedure}

The test site was located on a grass-covered field at the University of Kansas in Lawrence. The topographic slope was uniform at $<1 \%$. The surface material at the site is a rich, clayey soil layer $0.5-1.0 \mathrm{~m}$ thick. Below the soil layer is the Pennsylvanian Robbins Shale Member of the Lawrence Formation, extending to a depth of $25 \mathrm{~m}$ where a flat-lying limestone is encountered.

Presented at the 70th Annual Meeting, Society of Exploration Geophysicists. Manuscript received by the Editor March 14, 2000; revised manuscript received February 2, 2001.

${ }^{*}$ Formerly University of Kansas, Department of Geology, 120 Lindley Hall, Lawrence, Kansas 66045-2124; presently Jefferson County Geographic Information Systems, P. O. Box 447, Oskaloosa, Kansas 66066. E-mail: gis@ruralnet1.com.

$\ddagger$ The University of Kansas, Department of Geology, 120 Lindley Hall, Lawrence, Kansas 66045-2124. E-mail: kpilot@ukans.edu; dsteeples@ ukans.edu.

(C) 2001 Society of Exploration Geophysicists. All rights reserved. 
The data were collected using two parallel lines spaced $1 \mathrm{~m}$ apart (Figure 1). Each line consisted of $72 \mathrm{~L}-40 \mathrm{~A} 100-\mathrm{Hz}$ Mark Products geophones with a $15-\mathrm{cm}$ geophone interval. Geophones with $12.5-\mathrm{cm}$ spikes were planted in the ground by hand to prepare the control line. The test line consisted of 72 geophones bolted to four $2.7-\mathrm{m}$ lengths of 10 -cm-wide channel iron with 18 geophones per channel iron segment (Figure 2). The geophones mounted to the channel iron were screwed into 9.5 -mm (3/8-inch) NF-threaded bolts, $4 \mathrm{~cm}$ long (Figure 3). Next, the bolts were inserted into the channel iron through 10-mm drill holes and were fastened snugly with 9.5-mm NF-threaded nuts. Geophone spikes $12.5 \mathrm{~cm}$ long were then screwed onto the ends of the bolts. The four channel iron segments were not connected to each other on the ground during data acquisition. Two to three people stood on each piece of channel iron for about $5 \mathrm{~s}$ to push the attached geophone spikes into the ground.

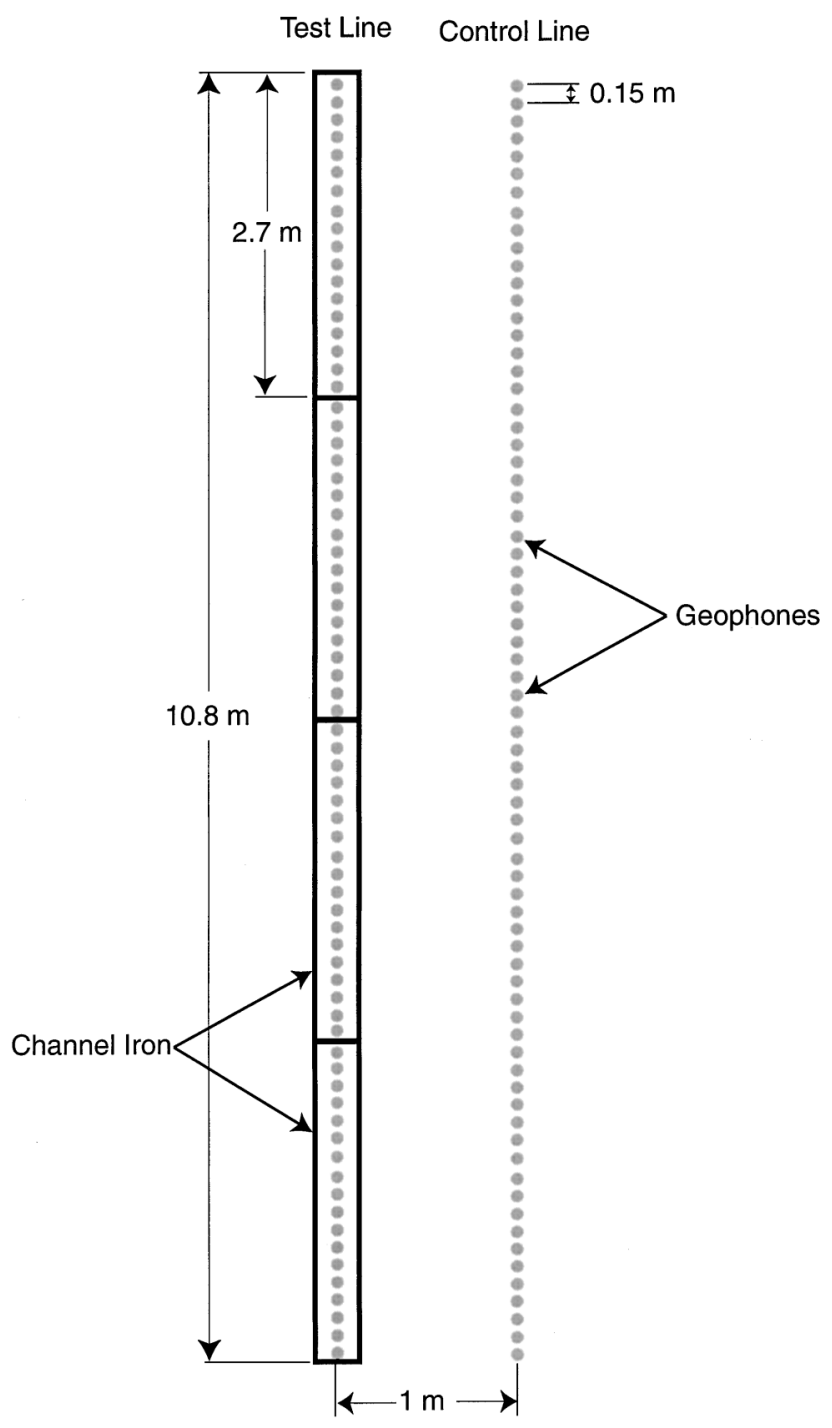

FIG. 1. Schematic of the field set-up for one field file. The parallel lines were $1 \mathrm{~m}$ apart. Each of the four segments of channel iron was $2.7 \mathrm{~m}$ long, with 18 geophones per bar at $15-\mathrm{cm}$ spacings. Pseudowalkaways from both lines were constructed using five 72-trace field files for each line. The 30.06 rifle source was shot every $10.8 \mathrm{~m}$ off the end of the test line.

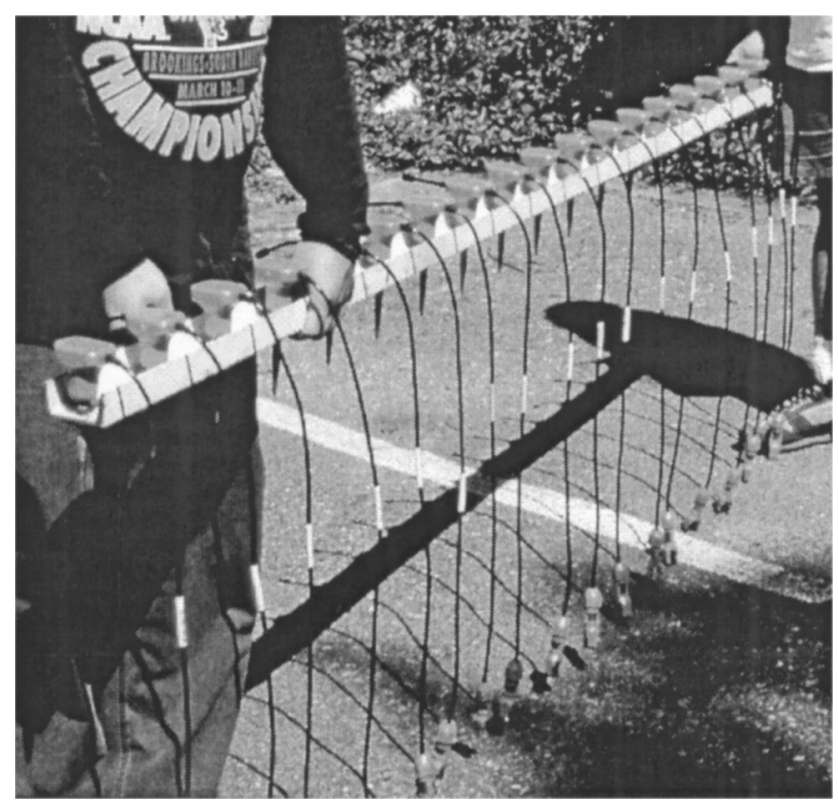

FIG. 2. Photograph of a channel iron segment with 18 geophones rigidly attached.

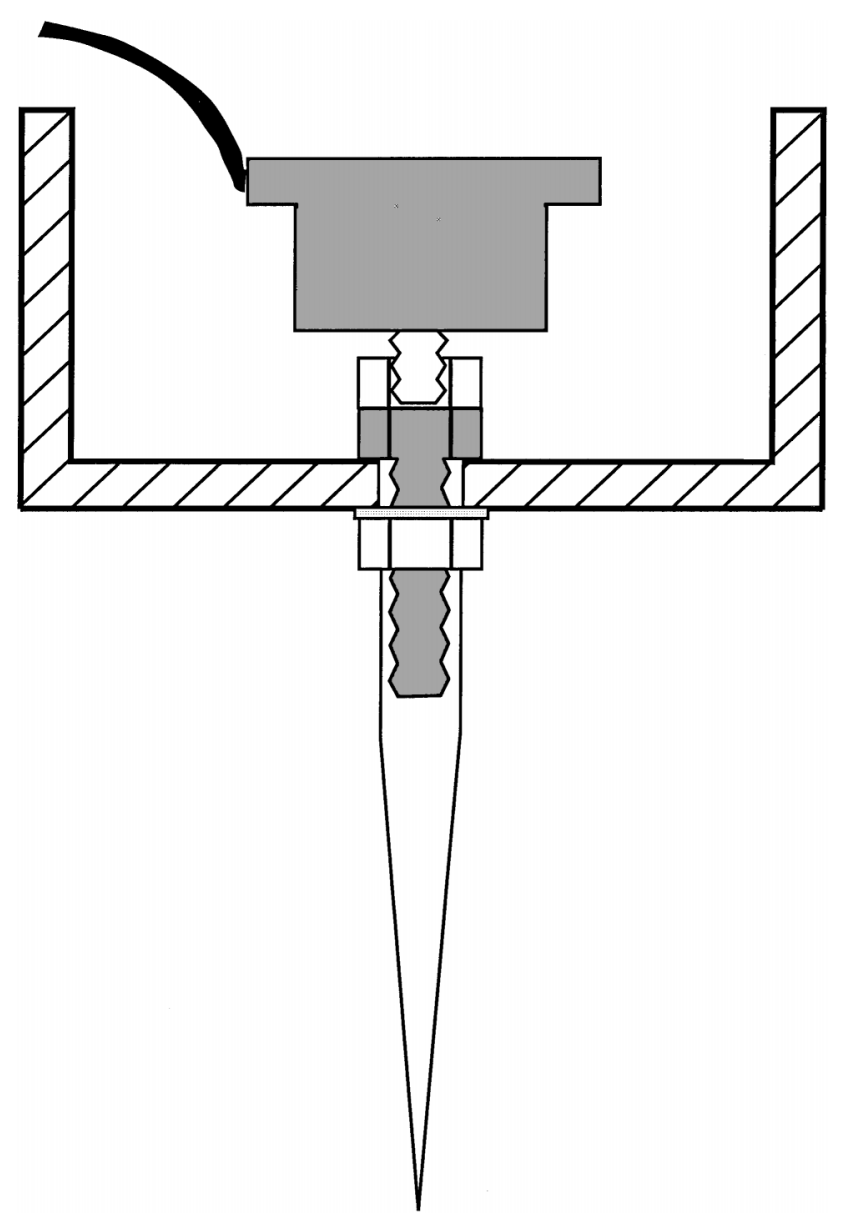

FIG. 3. Schematic cross-section of the rigidly mounted geophone/channel iron design (not to scale). The channel iron was $10 \mathrm{~cm}$ wide, with a $10-\mathrm{mm}$ hole drilled to accept a $9.5-\mathrm{mm}$ bolt. The upper $9.5-\mathrm{mm}$ NF nut was welded to the top of the bolt. 
The control-line data were collected using a 24-bit Bison 24096 seismograph with 72 channels. The data for the test line were recorded with a 72-channel, 24-bit Geometrics Strataview seismograph. Previous testing demonstrated that the difference in data because of instrument type between the two systems is negligible (Baker, 1999).

Figure 4 shows the control-line and test-line data. Each pseudowalkaway was constructed using five 72-channel field files. The pseudowalkaway test was acquired by fixing the geophone location of both the test and control lines and then moving the source away in 10.8-m increments. A 30.06 rifle was used as a seismic source in all of the field files generated. To maintain source consistency, the rifle was placed in prepunched holes $20 \mathrm{~cm}$ deep. Data were recorded using a sampling interval of $0.25 \mathrm{~ms}$, a $4-\mathrm{Hz}$ pre-A/D low-cut filter, and an antialias filter down $60 \mathrm{~dB}$ at $2000 \mathrm{~Hz}$.

\section{Experimental data}

Tests were conducted to determine whether the anomalous mode in Steeples et al. (1999a) could be detected when the

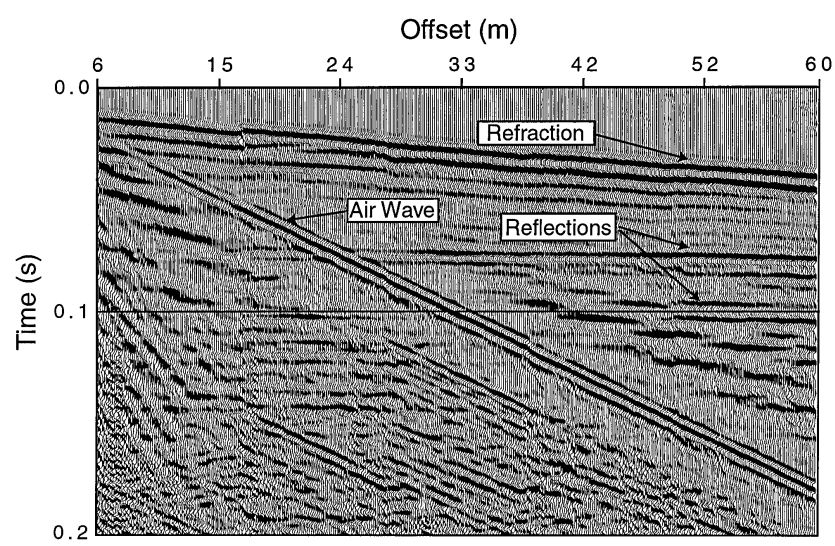

(a)

Pseudo-walkaway control-line data.

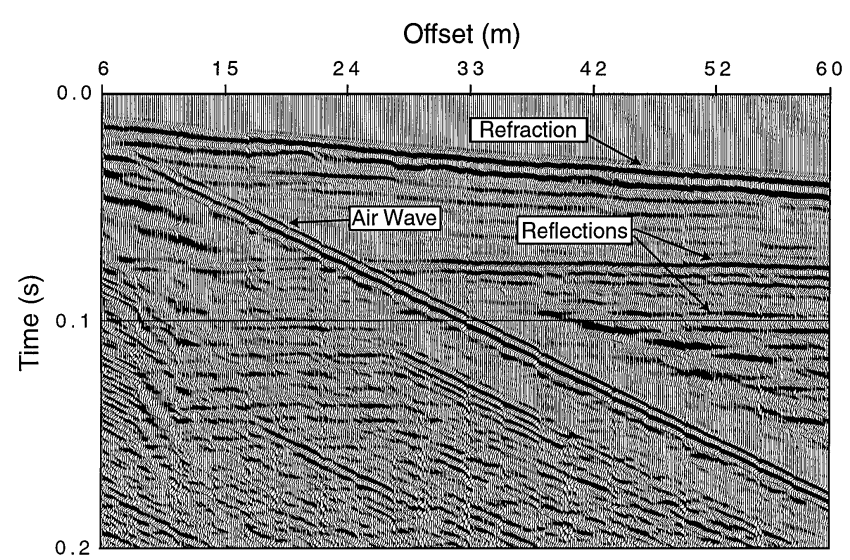

(b)

Pseudo-walkaway test-line data (channel iron)

FIG. 4. (a) Pseudowalkaway control-line data and (b) pseudowalkaway test-line data (channel iron). Data were obtained using the same shots. A comparison of Figure $4 \mathrm{a}$ with Figure $4 \mathrm{~b}$ shows no significant differences between the refracted and the reflected energy. The channel iron appears to have had no detrimental effect on the recorded data. channel iron pieces to which the geophones were attached were not connected rigidly to the tillage implement. The channel iron data collected during earlier testing (Steeples et al., 1999a), which revealed an undetermined interfering mode, used the same channel iron/geophone system as we used in this experiment. However, the channel iron to which the geophones were attached was rigidly coupled to a large farm implement, which was used to plant the geophones. After the geophones were planted, the implement remained attached to the channel iron. One possible source of the interfering mode noted in the Steeples et al. (1999a) data is the vibrational coupling of the farm implement to the geophones.

The data from both lines showed four dominant features: a $2000-\mathrm{m} / \mathrm{s}$ refraction, the airwave, and reflections at 72 and $94 \mathrm{~ms}$. A visual examination of the two pseudowalkaways in Figure 4 showed only minor discrepancies between the control line and the test line. Slight discontinuities were noted at intervals of $2.7 \mathrm{~m}$ between adjacent pieces of channel iron and every $10.8 \mathrm{~m}$ in the pseudowalkaway construction when the field-file plots were placed side by side. Similar discontinuities also appeared in the refraction and reflection modes. However, the extraneous vibrational mode in the Steeples et al. (1999a) data is not present in the data given in Figure 4.

Steeples et al. focus on the general extent to which seismic information could be detected by rigidly mounting geophones to a wooden board (1999b) and to channel iron (1999a). One of the primary goals of our experiment was to detect near-surface reflections when using channel iron-mounted geophones. Two reflections were found in the data-one at $72 \mathrm{~ms}$ and the other at $94 \mathrm{~ms}$ - at depths of about 90 and $120 \mathrm{~m}$, respectively. Comparing the reflections observable in each portion of Figure 4 shows no significant differences in arrival time or wavelet shape. The 72-ms reflection can be traced to offsets as small as $\sim 15 \mathrm{~m}$, even after the arrival of the airwave.

Frequency spectra for the control and test lines are shown in Figure 5. Each spectrum was calculated by averaging 20 individual frequency spectra formed from their associated whole

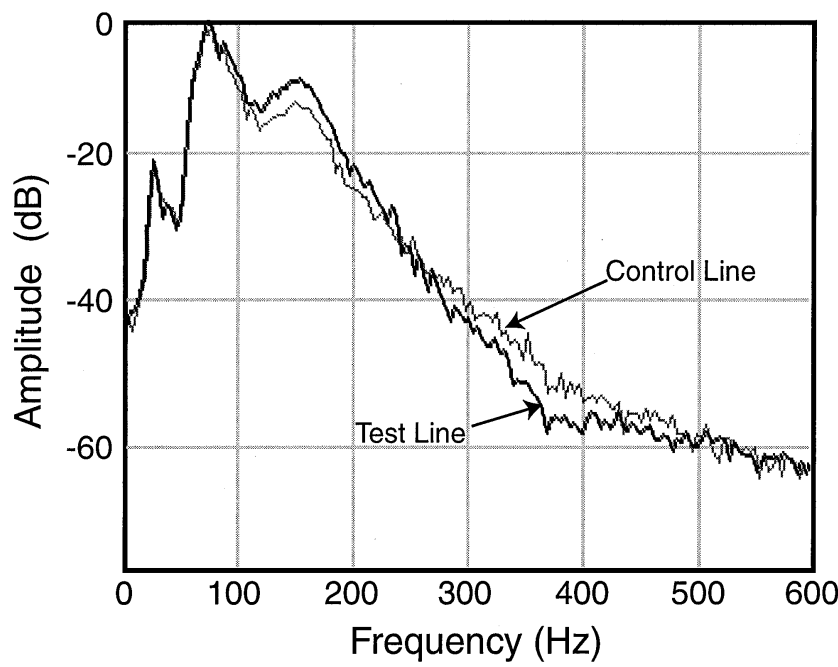

FIG. 5. Frequency spectra of the control line (light) and the test line (dark). The lines were planted $1 \mathrm{~m}$ apart. Each spectrum was created by averaging 20 individual frequency from their associated whole traces. The largest deviations $(\sim 4 \mathrm{~dB})$ occur at $\sim 150$ and $350 \mathrm{~Hz}$. 
traces, using offsets of $>30 \mathrm{~m}$. The overall trends of both spectra match quite well over a broad range of frequencies. However, two noticeable deviations in amplitude occur: one at about $150 \mathrm{~Hz}$ and a second at approximately $350 \mathrm{~Hz}$. Each deviation is approximately $4 \mathrm{~dB}$, which can be considered minor and is not necessarily attributable to the channel iron. Both the 72- and 94-ms reflections have dominant frequencies of $<150 \mathrm{~Hz}$ (i.e., $\sim 120 \mathrm{~Hz}$ ), so the drop in amplitude did not affect the frequency content of the reflections. Overall, the spectra show an excellent match between the two methods, with no indication that any extraneous modes were propagating in the channel iron.

To quantify the differences in individual traces between the test and control lines, normalized crosscorrelation was used on the first $100 \mathrm{~ms}$ of raw data (i.e., prior to the arrival of the airwave). Dividing the maximum crosscorrelation value (i.e., the value produced when the two traces matched best in time) by the geometric mean of the energies of the two traces gave the normalized correlation coefficient (Sheriff and Geldart, 1999).

First, five adjacent traces at offsets of 53.4 to $54.0 \mathrm{~m}$ were crosscorrelated in the control line to establish the extent to which traces within the same line correlated (Table 1). Correlation coefficients for the control line ranged from 0.985 to 0.995. The equivalent-offset traces from within the test line were then crosscorrelated, with all five traces from the test line connected to the same piece of channel iron. Correlation coefficients from within the test line were slightly higher, ranging from 0.992 to 0.996 .

Second, the five pairs of equivalent-offset traces from two lines were crosscorrelated to determine the degree to which individual traces were similar (Table 1). The correlation coefficients for interline comparisons ranged from 0.960 to 0.974 . We judged these relatively high coefficients to demonstrate near equivalence between the traces obtained by the geophones mounted on the channel iron and those planted normally.

For crosscorrelations within the two separate lines, the reference trace was $0.15 \mathrm{~m}$ closer than the correlation trace (Table 1 ). For the crosscorrelations from one line to the other, the testline trace was correlated to the control-line trace with the same offset.

\section{DISCUSSION AND CONCLUSIONS}

A comparison of Figures $4 a$ and $4 b$ shows high-quality refraction and reflection data from channel iron-mounted geo- phones. These compare well with the data recorded with conventionally planted geophones $1 \mathrm{~m}$ away. The high quality of the $P$-wave refractions observed is similar to previously recorded refraction data (Steeples et al., 1999a). No dominant interfering wave modes were found and, notably, two reflections are present.

Earlier studies (Steeples et al., 1999a) show that useful refraction data can be acquired when using geophones mounted on channel iron. However, whether some type of interfering mode was masking the later-arriving reflection information remained unclear. Figure 4 shows that high-quality, nearsurface seismic reflection data can be detected using the channel iron system. Qualitatively, the reflection information for both the test and control lines matches well. Reflected energy from the test line showed small changes in, but no degradation of, the reflected wavelet when compared to the control line, with no anomalous modes detected. The discontinuities present in the airwave, refraction, and reflections can be attributed to the end effects of the channel iron pieces. Included in those effects are modes that may originate in the channel iron. Possible rocking modes of the channel iron, the mass of the iron pieces slightly changing near-surface conditions, and different qualities of geophone plants may also contribute to the slight discontinuities.

Additionally, comparisons of the frequency spectra (0$600 \mathrm{~Hz}$ ) from the control and test lines failed to show any significant differences attributable to the rigid mounting of the geophones. Factors that may have affected the frequency content include slight variations in soil compaction and makeup, geophone plants that were not optimal and perhaps not exactly vertical, and buried roots from brush growing in the field.

Normalized crosscorrelation data showed that adjacent traces within the test line exhibited slightly more similarity than did traces within the normal plant control line. The correlation coefficients decreased by only about $1 \%$ when the control line and the test line were compared. Because of this small difference between the lines, we believe the geologic information that could be extracted from the two data sets is equivalent. Even though the spacing between the lines was only $1 \mathrm{~m}$, local variations in soil conditions noted during hand augering at the site may have contributed to the slight change in data quality.

The slightly lower correlation coefficients between the control line and the test line may indicate that the geophone plants in the test line were more nearly uniform. If the orientation of

Table 1. Normalized crosscorrelation traces and coefficients with line and offset.

\begin{tabular}{lccc}
\hline Crosscorrelation & Reference trace & Correlation trace & Coefficient \\
\hline Control line & CL-53.40 & CL-53.55 & 0.994 \\
& CL-53.55 & CL-53.70 & 0.995 \\
& CL-53.70 & CL-53.85 & 0.985 \\
Test line & CL-53.85 & CL-54.00 & 0.993 \\
& TL-53.40 & TL-53.55 & 0.996 \\
& TL-53.55 & TL-53.70 & 0.995 \\
Control/test lines & TL-53.70 & TL-53.85 & 0.994 \\
& TL-53.85 & TL-54.00 & 0.992 \\
& CL-53.40 & TL-53.40 & 0.974 \\
& CL-53.55 & TL-53.55 & 0.969 \\
& CL-53.70 & TL-53.70 & 0.969 \\
& CL-54.00 & TL-53.85 & 0.960 \\
\hline
\end{tabular}

$\mathrm{CL}=$ control line; $\mathrm{TL}=$ test line. 
the geophones attached to an entire segment of channel iron were not exactly vertical, then all of the geophone traces would still be consistent because their axes of motion were fixed parallel to each other. The drop in the correlation coefficients for the control line could be attributed in part to variations in the verticality of the geophone plants within the control line. Furthermore, the factors listed above that may have affected frequency content may have also affected these correlation coefficients.

These tests demonstrate that multiple, rigidly mounted geophones can be used to detect various propagation modes in the seismic wavefield. Some nonintuitive reasons concerning why this method works include the following. In the experiment, rigidly attached geophones were mounted on a $2.7-\mathrm{m}-$ long piece of channel iron with a maximum crosssectional dimension of about $10 \mathrm{~cm}$. The first arrivals would be expected to excite motion not only in the geophones but also in the channel iron. Physics dictates that compressional waves would propagate within the channel iron at a speed of about $5950 \mathrm{~m} / \mathrm{s}$ (Weast and Selby, 1967). The wave would be expected to propagate back and forth as a type of standing wave. However, no such wave was detected by the geophones. The lowest frequency (i.e., the longest wavelength) expected to propagate in the bar as a standing wave was estimated to be $1100 \mathrm{~Hz}$, when $\lambda / 2=2.7 \mathrm{~m}$ (the length of the bar) and the velocity of a $P$-wave in iron $=5950 \mathrm{~m} / \mathrm{s}$. Higher frequency energy would also be expected to propagate in the channel iron when $\lambda / 2<2.7 \mathrm{~m}$. However, at commonly observed seismic frequencies, we believe that the long wavelengths of any potentially detectable modes in the channel iron are likely to be large relative to the dimensions of the channel iron pieces.

Another potentially detectable mode in the data may be termed a flexural mode, which is not a wave but is the macroscopic flexing of the channel iron. The $P$ - and $S$-waves propagating in the channel iron are not the same as flexural modes. However, data acquired at this site did not show any flexural modes propagating through the channel iron pieces.

Our study addressed the recording of reflections using channel iron-mounted geophones and an anomalous interfering mode seen in the channel iron data of Steeples et al. (1999b) In endeavoring to isolate this mode, we detached the channel iron from the farm implement and then manually planted each 2.7-m piece of geophone-bearing channel iron in the ground. When this method was used, we obtained high-quality reflection information but did not detect the anomalous mode in the field data. This indicates the mode may have been generated by a vibrational coupling to or a rocking motion in the farm implement. Thus, depending on the method of automation, it may be necessary for the planted geophones to be detached from the planting mechanism during data acquisition and then reattached before they are moved to the next location. Alternatively, $f-k$ filtering may be able to remove the unwanted mode.

Clearly, a significant portion of the wavefield (direct waves, refractions, and reflections) can be detected by rigidly mounted geophones. Methods of this type may soon open the door to cost-effective ultrashallow 2-D and 3-D seismic surveys.

\section{ACKNOWLEDGMENTS}

This work was supported in part by the U.S. Department of Energy under contract DE-FG07-97-ER14826 and the National Science Foundation under grant EAR97061218.

\section{REFERENCES}

Baker, G. S., 1999, Seismic imaging shallower than three meters: Ph.D. dissertation, Univ. of Kansas.

Baker, G. S., Schmeissner, C., Steeples, D. W., and Plumb, R. G., 1999, Seismic reflections from depths of less than two meters: Geophys Res. Lett., 26, 279-282.

Baker, G. S., Steeples, D. W., Schmeissner, C., Pavlovic, M., and Plumb, R., 2001, Near-surface imaging using coincident seismic and GPR data: Geophys. Res. Lett., 28, 627-630.

Sheriff, R. E., and Geldart, L. P., 1999, Exploration seismology, 2nd ed.: Cambridge Univ. Press.

Steeples, D. W., Baker, G. S., and Schmeissner, C., 1999a, Toward the autojuggie: Planting 72 geophones in 2 sec: Geophys. Res. Lett., 26, 1085-1088.

Steeples, D. W., Baker, G. S., Schmeissner, C., and Macy, B. K., 1999b, Geophones on a board: Geophysics, 64, 809-818.

Weast, R. C., and Selby, S. M., Eds., 1967, Handbook of chemistry and physics, 47th ed.: The Chemical Rubber Co., Cleveland. 